\title{
Characterization of hepatocyte-based in vitro systems for reliable toxicity testing
}

\author{
Mathieu Vinken ${ }^{1} \cdot$ Jan G. Hengstler ${ }^{2}$
}

Received: 16 August 2018 / Accepted: 21 August 2018 / Published online: 23 August 2018

c) Springer-Verlag GmbH Germany, part of Springer Nature 2018

\begin{abstract}
A plethora of human hepatocyte-based in vitro systems for toxicity testing has been developed over the past few years. These systems are either directly derived from liver tissue or have been generated through stem cell technology. A wide variety of parameters is currently used to demonstrate the acquisition of in vivo-like hepatocellular physiology and toxicity in such novel in vitro systems. This frequently leads to flawed claims regarding applicability and may impede comparison between in vitro systems. A possible solution lies in defining a set of consensus criteria for proper benchmarking. A proposal for characterization of hepatocyte-based in vitro systems for toxicity screening is made in this paper and consists of testing critical features of viability, morphology, functionality and toxicity.
\end{abstract}

Keywords Hepatocyte $\cdot$ In vitro system $\cdot$ Viability $\cdot$ Morphology $\cdot$ Functionality $\cdot$ Toxicity

\section{Introduction}

Because of its unique anatomical localization and physiological function in the organism, the liver, and in particular the hepatocyte, represents a major target for systemic toxicity. For this reason, a lot of efforts have been focused, and are still being focused, on the development of hepatocyte-based in vitro systems eligible for the safety evaluation of chemical compounds (Fraczek et al. 2013; Hewitt et al. 2007; Leist et al. 2017). As a result, 2 main types of human hepatocyte-based in vitro systems are available today. The first are liver-derived systems that are directly obtained from liver or liver tissue. They vary in complexity and longevity, and include whole isolated perfused livers, liver slices, primary hepatocytes and their diverse culture configurations, cell lines and subcellular liver fractions (Fraczek et al. 2013; Godoy et al. 2013). Among those, adherent cultures of primary human hepatocytes are still considered as the

Mathieu Vinken

mvinken@vub.ac.be

1 Department of In Vitro Toxicology and Dermato-Cosmetology, Vrije Universiteit Brussel, Laarbeeklaan 103, 1090 Brussels, Belgium

2 Leibniz Research Centre for Working Environment and Human Factors at the Technical University of Dortmund (IfADo), Dortmund, Germany gold standard. Nevertheless, although a multitude of studies published in the past decades has demonstrated the potential of a variety of primary hepatocyte culture formats to predict different types of chemical-induced hepatotoxicity, they cope with progressive deterioration of their in vivo-like functional and morphological features (Fraczek et al. 2013). This dedifferentiation process hampers the use of primary hepatocyte cultures for testing toxicity over longer terms (Godoy et al. 2016). Furthermore, human liver tissue for the isolation of primary hepatocytes and thus for in vitro modelling purposes is rather scarce. The second class of hepatocyte-based in vitro systems are hepatic cells differentiated from stem cells of different origin, including human embryonic and adult stem cells (Godoy et al. 2015, 2018). The induced pluripotent stem cell technique has undoubtedly gained most attention in this respect. However, despite being promising, this procedure could still benefit from improvement, as the resulting hepatocyte-like cells are not able to pick up all types of liver insults triggered by chemical compounds (Bell et al. 2017).

As holds for science in general, the field of in vitro toxicology has evolved to become a multidisciplinary area over the past decade. Indeed, by synergizing expertise from relevant disciplines, including toxicology, hepatology, bio-engineering, cell biology and immunology, several cutting-edge liver-derived and stem cell-derived in vitro systems have been introduced lately. These systems allow, at least in part, 
to tackle challenges like dedifferentiation or poor toxicological responses to chemicals. Such interdisciplinary endeavours should be strongly encouraged, as they will expedite the establishment of reliable in vitro systems that accurately predict chemical-induced hepatotoxicity (Ghallab et al. 2016). A wide spectrum of parameters is currently used to demonstrate the acquisition of in vivo-like hepatocellular physiology and toxicity in such novel hepatic in vitro systems. This often leads to merely partially valid or even invalid claims regarding applicability and may hinder sound comparison between in vitro systems. This could be overcome by harmonizing and defining a number of performance criteria. A proposal for such optimal characterization that will enable objective benchmarking of hepatocyte-based in vitro systems for toxicity testing purposes is made here below. The proposed characterization exercise consists of testing critical elements of viability, morphology, functionality and toxicity.

\section{Viability}

In normal liver, the incidence of spontaneous apoptosis is very low, affecting only $0.05-0.1 \%$ of all liver cells and particularly taking place in hepatocytes in the perivenous acinar region (Qiao and Farrell 1999). However, in several acute and chronic liver diseases as well as during chemicalinduced hepatotoxicity, apoptotic activity strongly increases (St-Pierre and Dufour 2012). This also holds true when setting up primary hepatocyte cultures. Nevertheless, apoptosis is not the sole mode of cell death that underlies cell demise in primary hepatocyte cultures. In fact, 2 peaks of cell death can be discerned in this in vitro setting, both of which involve apoptosis and necrosis. The cell death wave during early time points of cultivation reflects cell damage caused following the hepatocyte isolation procedure. When the cell culture medium is renewed on a daily basis, and thus defunct hepatocytes are removed, apoptotic and necrotic biomarkers reach minimal values around the second day of cultivation. Subsequently, a second cell death peak is noticed, which probably is a manifestation of the poor and unfavourable accommodation of the hepatocytes to the artificial cultivation conditions and hence the absence of the vital in vivo micro-environment (Vinken et al. 2011).

Overall, a constant baseline viability of at least $90 \%$ is required to distinguish spontaneous cell demise from cell death induced by toxicants to be tested in hepatocyte-based in vitro systems. A pivotal determinant of spontaneous cell death in vitro is cell density. In this respect, hepatocytes plated at low density $\left(0.35 \times 10^{5}\right.$ cells per $\left.\mathrm{cm}^{2}\right)$ display less cell death than their counterparts seeded at high density $\left(1.4 \times 10^{5}\right.$ cells per $\left.\mathrm{cm}^{2}\right)$, which is about half of the in vivo density in liver $\left(2-3 \times 10^{5}\right.$ cells per $\mathrm{cm}^{2}$ ) (Qiao and Farrell 1999). However, unlike cell survival, cell density positively correlates with the functional status of the cultured hepatocytes (Hamilton et al. 2001).

The choice of the viability assay requires special attention. Numerous cell viability assays are available, all that rely on various parameters to monitor viability as such, and therefore, that detect cytotoxicity with different sensitivities and specificities. For this reason, it may be an advantage to combine 2 assays that assess early and late key events of cytotoxicity, such as compromised metabolic activity and damage to the cell plasma membrane surface, respectively. A typical and reliable combination of viability assays includes the [3-(4,5-dimethylthiazol-2-yl)-2,5-diphenyltetrazolium bromide] test and the lactate dehydrogenase leakage test (Vinken and Blaauboer 2017).

\section{Morphology}

In vivo, hepatocytes are highly polarized polygonal cells with a diameter of $20-25 \mu \mathrm{m}$, of which at least $20 \%$ are binucleated. Upon isolation, primary hepatocytes lose all intercellular connections and polarity, resulting in rounded cells. When seeded under conventional culture conditions, hepatocytes adhere to the plastic surface of the cell culture dish within $4 \mathrm{~h}$ and aggregate in groups of 2-10 cells to re-establish intercellular contacts. After $12 \mathrm{~h}$ of cultivation, hepatocytes start to regain their typical polyhedral morphology, and, finally, after 1 day, bile canaliculi are reconstituted and de novo synthesized intercellular junctions appear (Wanson et al. 1977). Upon dedifferentiation, hepatocytes adopt a fibroblast-like flattened phenotype. Hepatocytes either swell and become leaky, or shrink and produce apoptotic bodies when dying by necrosis or apoptosis, respectively (Fraczek et al. 2013; Vinken et al. 2014).

Although cell architecture not always fully reflects functionality, which is particularly true for stem cell-derived systems, it is advisable to monitor cellular morphology in hepatocyte-based in vitro systems. This can be done in first instance by means of regular light microscopy. Electron microscopy allows more in-depth characterization, including detection of structural polarity markers, such as microvilli and tight junctions in the apical area, but is not practicable for the control of individual batches in everyday routine. Immunocytochemistry analysis may be used concomitantly for in situ staining of polarity indicators at the sinusoidal/basolateral pole, like the asialoglycoprotein receptor, the sodium-potassium adenosine 5'-triphosphatase and the sodium-taurocholate cotransporter, as well as at the apical pole, including dipeptidyl peptidase IV, gamma glutamyltranspeptidase and the bile salt export pump. Furthermore, immunohistochemistry analysis of markers of mature hepatocyte cyto-architecture, such as the intermediate filament cytokeratin 18, can be performed (Gissen and Arias 
2015; Treyer and Müsch 2013). Clearly, the most convincing evidence relates to functional polarity (Deharde et al. 2016). This includes testing of the functionality of apical drug transporters using fluorescent bile salt analogues, such as cholyl-L-lysyl-fluorescein or 5-chloromethylfluorescein diacetate that is taken up by hepatocytes. Fluorescence is hereby generated by intracellular esterase hydrolysis followed by excretion into the bile canaliculi (Reif et al. 2015). Also, tracing intramembrane diffusion of fluorescent lipid probes or measuring transepithelial electrical resistance are widely used methods to test tight junction functionality, in particular the junctional fence and paracellular gate, respectively (Matter and Balda 2003). Of more pharmaco-toxicological relevance are drug transporter assays, which offer the advantage to combine testing of polarity and hepatocellular functionality. In this respect, functionality testing of the sinusoidal sodium-taurocholate cotransporter, the apical bile salt export pump and multidrug resistance protein 2, using fluorescent probes and inhibitors, is a possible suggestion (Ramboer et al. 2013; Yang et al. 2016).

\section{Functionality}

The liver performs a number of vital functions, such as metabolism of carbohydrates and lipids, bile acid production and transport, secretion of albumin and blood coagulation factors, as well as the detoxification of endobiotics and xenobiotics. Most of these functions are carried out by hepatocytes (Fraczek et al. 2013). At the most upstream regulatory level of gene expression, the production of the mediators of hepatocyte functionality is controlled by the orchestrated interplay between liver-enriched transcription factors and nuclear receptors (Lau et al. 2018). Accordingly, demonstration of hepatocyte functionality could start by testing expression of these liver-enriched transcription factors, such as CCAAT/enhancer-binding protein alpha, hepatocyte nuclear factors 1 alpha and 4 alpha, and nuclear receptors, like the constitutive androstane receptor and the pregnane $\mathrm{X}$ receptor, as well as of the functionality mediators as such. The latter typically are biotransformation enzymes (Schrem et al. 2002, 2004). It should be stressed, however, that not only prominent phase I biotransformation enzymes, including cytochrome P450 1A2, 2B6, 2C9, 2C19, 2D6, 2E1 and $3 \mathrm{~A} 4$, should be detected, but equally important phase II biotransformation enzymes, such as those belonging to the uridine diphosphate-glucuronosyltransferase, glutathione $S$-transferase and sulfotransferase families (Grinberg et al. 2014). Since drug transport is also considered an essential part of xenobiotic metabolism, the expression of effectors of this critical process, with sodium-taurocholate cotransporter, the bile salt export pump and multidrug resistance protein 2 as prototypes, should also be investigated (Ramboer et al.
2013). Because mRNA is not always translated into the corresponding protein, it is advisable to study expression of these markers also by immunocytochemistry or immunoblot analysis rather than merely by microarray analysis or real-time reverse transcriptase polymerase chain reaction. Furthermore, sound characterization of hepatocyte-based in vitro systems cannot be restricted to expression analysis, but should also include functional analysis. In this context, several assays, based on the use of substrates, probes and inhibitors, are currently available to test the activity of the abovementioned biotransformation and drug transport mediators. An important aspect includes demonstration of inducibility of these mediators by applying well-established in vivo-relevant drug-based inducers. Additional hepatocytespecific functions, such as albumin secretion, ammonia detoxification and glycogen storage, should also be preferably assessed at the functional level in parallel.

\section{Toxicity}

In view of using hepatocyte-based in vitro systems for predicting adverse effects induced by chemicals, it is important to confirm their capacity to detect prototypical types of liver toxicity. Type A or intrinsic types of drug-induced liver injury, which are predictable and reproducible in nature, are adequate for this purpose (Vinken et al. 2013a). A first classical case includes acetaminophen-related liver toxicity. The mechanism of this type toxicity involves saturation of glucuronidation and sulfation biotransformation pathways and concomitant conversion, partly mediated by cytochrome P450 2E1, to a toxic metabolite that causes dose-dependent necrosis. Therefore, the manifestation of acetaminophenrelated hepatotoxicity, which can be monitored in vitro using necrosis markers, depends on the metabolic competence of the hepatocyte-based cell culture system (Maes et al. 2016). A second type of liver toxicity to be tested includes steatosis. Several drugs, such as valproic acid, induce lipid accumulation in hepatocytes, which can be detected by in vitro lipid staining. Valproic acid mainly triggers microvesicular steatosis by impairment of mitochondrial beta oxidation and inhibition of carnitine palmitoyl transferase 1. Of note, valproic acid undergoes bio-activation, supported in part by cytochrome $\mathrm{P} 4502 \mathrm{C}$, to a metabolite that is more steatogenic and cytotoxic compared to the parent drug (Willebrords et al. 2015). As for acetaminophen, valproic acid-related toxicity therefore necessitates high biotransformation capacity of the hepatocyte-based in vitro system. An alternative to steatosis as an adverse effect to be included during toxicological characterization of hepatocyte-based in vitro systems is phospholipidosis. However, the toxicological relevance of this type of effect is still under debate (Vinken et al. 2013a). A third type of liver toxicity to be 
tested includes cholestasis. Cholestatic liver injury is the result of a direct deteriorative response and an indirect adaptive response of hepatocytes to various insults, including those caused by drugs (Jansen et al. 2017). A common protocol to evoke cholestatic hepatotoxicity in vitro, which can be detected by chromatographic bile acid analysis, is the simultaneous exposure of hepatocyte-based cell culture systems to a cholestatic drug and a concentrated bile acid mixture. A first-choice drug in this respect is cyclosporine A, which induces cholestasis by inhibiting drug transporters, including the bile salt export pump, by compromising hepatocellular cyto-architecture and by affecting bile canalicular dynamics, all that can be monitored in vitro (Vinken et al. 2013b).

\section{Comparison to in vivo relevant concentrations}

As described in the previous paragraph, control compounds, such as acetaminophen or valproic acid, should cause positive test results in a hepatocyte-based in vitro system. Importantly, positive effects, such as cytotoxicity or lipid droplet accumulation, should occur at concentrations relevant to the human in vivo situation. For example, total human plasma peak concentrations of 1-2 $\mathrm{mM}$ acetaminophen are known to cause an increased risk of hepatotoxicity (Slattery and Levy 1979; Waring and Benhalim 2008). Therefore, this concentration range of acetaminophen should show a positive effect if an in vitro system is adequate. If much higher concentrations, such as $15 \mathrm{mM}$ as reported in some publications, are required to obtain cytotoxicity, the applicability of this hepatocyte-based in vitro system should be considered with caution. In contrast, lower plasma peak concentrations of acetaminophen, such as $40 \mu \mathrm{M}$, do not cause any increased risk of hepatotoxicity in vivo and should therefore also test negative in vitro.

Although this example seems straightforward, it remains a complex challenge to evaluate the in vivo relevance of a hepatocyte-based in vitro system. First, it is not trivial to decide to which pharmacokinetic parameters the concentrations used in an in vitro system should be compared. Often the total plasma peak concentration is used for this purpose (Godoy et al. 2013; Proctor et al. 2017). Based on the experience of the authors, this is probably not an unreasonable strategy. However, systematic studies comparing a set of possible pharmacokinetic parameters, like total versus free concentrations, or total plasma peak concentrations versus steady-state or average concentrations, are not yet available. Moreover, it became possible to not only model concentrations in mixed venous blood, but also in blood of the portal vein (Mielke et al. 2011), which may be used as a reference. Second, it should be considered that the intracellular concentrations of test compounds, and less the concentrations in blood or in cell culture medium, are critical for toxicity. Comparing concentrations in blood or plasma to those in the cell culture medium is based on the assumption that the ratios of test compounds in plasma to hepatocytes in vivo are similar to the ratios of cell culture medium concentrations to hepatocytes in vitro. Although this assumption may not be unreasonable, only little data are available. Third, human positive and negative control compounds lead to a further challenge. Human toxicity information is frequently available for only 1 exposure scenario, typically the therapeutic dosis. For 1 therapeutic schedule, such as 2 times $200 \mathrm{mg}$ per day for 3 weeks, human pharmacokinetics are usually precisely known together with the information whether this dosing scheme leads to an increased risk of hepatotoxicity or whether it is harmless. However, for non-hepatotoxic compounds, it is usually unknown whether already twofold higher or only 2000 -fold higher concentrations would lead to hepatotoxicity, because experience is available only for the therapeutic dosis. Vice versa, for hepatotoxic compounds, it usually remains unknown whether already twofold or 2000fold lower than therapeutic dosis would be non-toxic. In this context, the case of acetaminophen represents an unusual exception because it is well-documented that doses up to $4 \mathrm{~g}$ are not hepatotoxic in adults, while higher concentrations, for instance $10 \mathrm{~g}$, usually cause hepatotoxicity, a fact only known from the high number of accidental or suicidal intoxications. Therefore, it is relatively easy to test the response of hepatocyte-based in vitro systems to few well-characterized positive control compounds, such as acetaminophen. However, a systematic interpretation of hepatocyte-based in vitro systems with respect to their meaning for the human in vivo situation remains a major challenge for future research.

\section{Conclusion}

Objective evaluation of the performance, applicability and relevance of newly introduced hepatocyte-based in vitro systems is often problematic. A possible solution lies in a set of consensus criteria for benchmarking purposes. In this respect, a proposal for characterization of hepatocytebased in vitro systems for toxicity screening could consist of testing critical viability, morphology, functionality and toxicity features (Table 1). While doing so, the human hepatic in vivo situation obviously is the ideal standard for comparison. However, given the frequent lack of in vivo data and because of in vitro-in vivo extrapolation artefacts in general, freshly isolated or cryopreserved primary human hepatocytes are typically used as a standard when characterizing novel human hepatocyte-based in vitro systems. The use of 1-day-old primary human hepatocytes is less preferred, because functional dedifferentiation already manifests during the first few hours following hepatocyte 
Table 1 Parameters for the characterization of hepatocytebased in vitro systems for reliable toxicity testing

\author{
Viability: at least $90 \%$ \\ Metabolic competence analysis \\ Plasma membrane integrity analysis \\ Morphology: polygonal and polarized \\ Light and/or electron microscopic morphological analysis \\ In situ staining analysis of polarity markers and hepatocellular architecture \\ Functionality analysis of polarity markers \\ Functionality: similar to freshly isolated primary human hepatocytes \\ Protein expression analysis of liver-enriched transcription factors and nuclear receptors \\ Protein expression analysis of phase I and II biotransformation enzymes \\ Protein expression analysis of drug transporters \\ Functionality analysis of phase I and II biotransformation enzymes \\ Functionality analysis of drug transporters \\ Functionality analysis of albumin secretion, ammonia detoxification and glycogen storage \\ Toxicity: reproducing human in vivo intrinsic drug-induced liver injury \\ Acetaminophen-induced necrosis \\ Valproic acid-induced steatosis \\ Cyclosporine A-induced cholestasis
}

isolation (Fraczek et al. 2013). Similarly, defunct liver cell lines, like human hepatocellular carcinoma HepG2 cells, should not be used as controls (Hart et al. 2010). In many novel hepatocyte-based in vitro systems, hepatocytes are co-cultured together with other relevant hepatic cell types, such as stellate cells, which are either directly derived from liver or that have been differentiated from stem cell sources. Such heterocellular systems may be necessary to model specific types of toxicity, including fibrosis (van Grunsven 2017). It is clear that the performance of these non-hepatocellular liver cells also needs to be monitored to secure sound reflection of the in vivo situation, thereby using specific morphological and functional criteria as well as appropriate standards. Even in case of meeting all performance criteria, the in vitro setting may not fully guarantee to predict all types of toxicity. This is specifically valid for idiosyncratic types of drug-induced liver injury, which are difficult to study in vitro. In addition, non-hepatic factors, like the gut microbiota or the presence of pro-inflammatory factors, have been identified in the last few years as major determinants in the manifestation of chemical-induced liver toxicity (Ballet 2015). Such factors should be included in future liver-based in vitro systems and are anticipated to influence hepatocyte functionality and toxicity responses. Such innovations may require adaptation of performance criteria of hepatic in vitro systems.

Acknowledgements This work was supported by the grants of the European Research Council (ERC), the Fund for Scientific Research Flanders-Belgium (FWO), the University Hospital of the Vrije Universiteit Brussel-Belgium (Willy Gepts Fonds UZ-VUB) and the Center for Alternatives to Animal Testing (CAAT) at Johns Hopkins University Baltimore-USA.

\section{References}

Ballet F (2015) Preventing drug-induced liver injury: how useful are animal models? Dig Dis 33:477-485

Bell CC, Lauschke VM, Vorrink SU, Andersson TB, IngelmanSundberg M (2017) Transcriptional, functional, and mechanistic comparisons of stem cell-derived hepatocytes, HepaRG cells, and threedimensional human hepatocyte spheroids as predictive in vitro systems for drug-induced liver injury. Drug Metab Dispos 45:419-429

Deharde D, Schneider C, Hiller T, Zeilinger K, Damm G (2016) Bile canaliculi formation and biliary transport in 3D sandwich-cultured hepatocytes in dependence of the extracellular matrix composition. Arch Toxicol 90:2497-2511

Fraczek J, Bolleyn J, Vanhaecke T, Rogiers V, Vinken M (2013) Primary hepatocyte cultures for pharmaco-toxicological studies: at the busy crossroad of various anti-dedifferentiation strategies. Arch Toxicol 87:577-610

Ghallab A, Cellière G, Henkel SG, Gebhardt R, Hengstler JG (2016) Model-guided identification of a therapeutic strategy to reduce hyperammonemia in liver diseases. J Hepatol 64:860-871

Gissen P, Arias IM (2015) Structural and functional hepatocyte polarity and liver disease. J Hepatol 63:1023-1037

Godoy P, Hewitt NJ, Albrecht U, Yarborough KM, Hengstler JG (2013) Recent advances in 2D and 3D in vitro systems using primary hepatocytes, alternative hepatocyte sources and non-parenchymal liver cells and their use in investigating mechanisms of hepatotoxicity, cell signaling and ADME. Arch Toxicol 87:1315-1530

Godoy P, Schmidt-Heck W, Natarajan K, Hay DC, Hengstler JG (2015) Gene networks and transcription factor motifs defining the differentiation of stem cells into hepatocyte-like cells. J Hepatol 63:934-942

Godoy P, Widera A, Schmidt-Heck W, Dooley S, Hengstler JG (2016) Gene network activity in cultivated primary hepatocytes is 
highly similar to diseased mammalian liver tissue. Arch Toxicol 90:2513-2529

Godoy P, Schmidt-Heck W, Hellwig B, Blüthgen N, Hengstler JG (2018) Assessment of stem cell differentiation based on genomewide expression profiles. Philos Trans R Soc Lond B Biol Sci 373:20170221

Grinberg M, Stöber RM, Edlund K, Rahnenführer J, Hengstler JG (2014) Toxicogenomics directory of chemically exposed human hepatocytes. Arch Toxicol 88:2261-2287

Hamilton GA, Jolley SL, Gilbert D, Coon DJ, Barros S, LeCluyse EL (2001) Regulation of cell morphology and cytochrome P450 expression in human hepatocytes by extracellular matrix and cellcell interactions. Cell Tissue Res 306:85-99

Hart SN, Li Y, Nakamoto K, Subileau EA, Steen D, Zhong XB (2010) A comparison of whole genome gene expression profiles of HepaRG cells and HepG2 cells to primary human hepatocytes and human liver tissues. Drug Metab Dispos 38:988-994

Hewitt NJ, Lechón MJ, Houston JB, Groothuis GM, Hengstler JG (2007) Primary hepatocytes: current understanding of the regulation of metabolic enzymes and transporter proteins, and pharmaceutical practice for the use of hepatocytes in metabolism, enzyme induction, transporter, clearance, and hepatotoxicity studies. Drug Metab Rev 39:159-234

Jansen PL, Ghallab A, Vartak N, Hampe J, Hengstler JG (2017) The ascending pathophysiology of cholestatic liver disease. Hepatology 65:722-738

Lau HH, Ng NHJ, Loo LSW, Jasmen JB, Teo AKK (2018) The molecular functions of hepatocyte nuclear factors: in and beyond the liver. J Hepatol 68:1033-1048

Leist M, Ghallab A, Graepel R, van de Water B, Hengstler JG (2017) Adverse outcome pathways: opportunities, limitations and open questions. Arch Toxicol 91:3477-3505

Maes M, Vinken M, Jaeschke H (2016) Experimental models of hepatotoxicity related to acute liver failure. Toxicol Appl Pharmacol 290:86-97

Matter K, Balda MS (2003) Functional analysis of tight junctions. Methods 30:228-234

Mielke H, Anger LT, Schug M, Hengstler JG, Stahlmann R, GundertRemy U (2011) A physiologically based toxicokinetic modelling approach to predict relevant concentrations for in vitro testing. Arch Toxicol 85:555-563

Proctor WR, Foster AJ, Vogt J, Messner S, Williams D (2017) Utility of spherical human liver microtissues for prediction of clinical drug-induced liver injury. Arch Toxicol 91:2849-2863

Qiao L, Farrell GC (1999) The effects of cell density, attachment substratum and dexamethasone on spontaneous apoptosis of rat hepatocytes in primary culture. In Vitro Cell Dev Biol Anim $35: 417-424$

Ramboer E, Vanhaecke T, Rogiers V, Vinken M (2013) Primary hepatocyte cultures as prominent in vitro tools to study hepatic drug transporters. Drug Metab Rev 45:196-217
Reif R, Karlsson J, Günther G, Beattie L, Hengstler W, Jirstrand JG M (2015) Bile canalicular dynamics in hepatocyte sandwich cultures. Arch Toxicol 89:1861-1870

Schrem H, Klempnauer J, Borlak J (2002) Liver-enriched transcription factors in liver function and development. Part I: the hepatocyte nuclear factor network and liver-specific gene expression. Pharmacol Rev 54:129-158

Schrem H, Klempnauer J, Borlak J (2004) Liver-enriched transcription factors in liver function and development. Part II: the C/EBPs and D site-binding protein in cell cycle control, carcinogenesis, circadian gene regulation, liver regeneration, apoptosis, and liverspecific gene regulation. Pharmacol Rev 56:291-330

Slattery JT, Levy G (1979) Acetaminophen kinetics in acutely poisoned patients. Clin Pharmacol Ther 25:184-195

St-Pierre MV, Dufour JF (2012) Biomarkers for hepatocellular apoptosis in the management of liver diseases. Curr Pharm Biotechnol 13:2221-2227

Treyer A, Müsch A (2013) Hepatocyte polarity. Compr Physiol 3:243-287

van Grunsven LA (2017) 3D in vitro models of liver fibrosis. Adv Drug Deliv Rev 121:133-146

Vinken M, Blaauboer BJ (2017) In vitro testing of basal cytotoxicity: establishment of an adverse outcome pathway from chemical insult to cell death. Toxicol In Vitro 39:104-110

Vinken M, Decrock E, Doktorova T, Leybaert L, Rogiers V (2011) Characterization of spontaneous cell death in monolayer cultures of primary hepatocytes. Arch Toxicol 85:1589-1596

Vinken M, Maes M, Vanhaecke T, Rogiers V (2013a) Drug-induced liver injury: mechanisms, types and biomarkers. Curr Med Chem 20:3011-3021

Vinken M, Landesmann B, Goumenou M, Whelan M, Rogiers V (2013b) Development of an adverse outcome pathway from drug-mediated bile salt export pump inhibition to cholestatic liver injury. Toxicol Sci 136:97-106

Vinken M, Maes M, Oliveira AG, Vanhaecke T, Rogiers V (2014) Primary hepatocytes and their cultures in liver apoptosis research. Arch Toxicol 88:199-212

Wanson JC, Drochmans P, Mosselmans R, Ronveaux MF (1977) Adult rat hepatocytes in primary monolayer culture: ultrastructural characteristics of intercellular contacts and cell membrane differentiations. J Cell Biol 74:858-877

Waring WS, Benhalim S (2008) Serum acetaminophen concentrations after acute overdose are not altered by opioid co-ingestion. J Toxicol Sci 33:549-553

Willebrords J, Pereira IV, Maes M, Cogliati B, Vinken M (2015) Strategies, models and biomarkers in experimental non-alcoholic fatty liver disease research. Prog Lipid Res 59:106-125

Yang K, Guo C, Woodhead JL, Howell BA, Brouwer KLR (2016) Sandwich-cultured hepatocytes as a tool to study drug disposition and drug-induced liver injury. J Pharm Sci 105:443-459 